\title{
Fruit juice of Garcinia indica Choisy modulates dyslipidemia and lipid metabolism in cafeteria diet based rat model
}

\author{
Laxmipriya Nampoothiri», Prashant Sudra, Arpi Dey, Shivani Dhadhal, Azazahemad A. Kureshi***, Satyanshu Kumar**, \\ Tushar Dhanani**, Raghuraj Singh** and Premlata Kumari* \\ Department of Biochemistry, Faculty of Science, The Maharaja Sayajirao University of Baroda, Vadodara-390002, Gujarat, India \\ *Department of Chemistry, Sardar Vallabhbhai National Institute of Technology, Surat-395007, Gujarat, India \\ **ICAR-Directorate of Medicinal and Aromatic Plants Research, Boriavi-387310, Gujarat, India
}

\section{Article Info}

\section{Article history}

Received 3 May 2021

Revised 20 June 2021

Accepted 21 June 2021

Published online 30 June 2021

\section{Keywords}

Garcinia indica Choisy

Dyslipidemia

Obesity

Hydroxycitric acid

HPLC

\begin{abstract}
There is a significant rise in the incidences of dyslipidemia, leading to obesity. The therapeutics available for dyslipidemia are limited and associated with major side-effects. Thereby, researchers are shifting towards nutraceuticals compounds. In the current study, Garcinia indica Choisy, which is an endemic species of Western Ghats of India was evaluated for its anti-dyslipidemic properties in cafeteria diet fed obese rat model. Firstly, cafeteria diet fed rat model was developed and validated. After successful development of the model, the rats were orally fed with $1 \mathrm{ml} \mathrm{G}$. indica fruit juice for 4 weeks and parameters such as OGTT, lipid profile, hormone levels of insulin and leptin, HMG CoA reductase and LCAT enzyme activities and toxicity parameters were evaluated. Identification and quantification of the hydroxycitric acid in $G$. indica fruit juice was done by HPLC method. Toxicity parameters like SGPT and creatinine were performed to evaluate the toxicity of the dose. Results showed that cafeteria diet fed animals exhibited increased body weight, increased food intake, decreased water intake, increased glucose intolerance and dyslipidemia at 10 weeks. Treatment with $G$. indica fruit juice for 4 weeks, reduced the body weight, improved the metabolic parameters like glucose sensitivity, dyslipidemia, insulin and leptin levels and lipid metabolizing levels without causing toxicity. Oral dosage of $G$. indica fruit juice for 4 weeks exhibits antiobesity potential in cafeteria diet fed dyslipidemic rats. The results obtained were better than orlistat, which is a standard mode of chemotherapy for management of dyslipidemic obesity.
\end{abstract}

\section{Introduction}

The prevalence of dyslipidemia leading to obesity is rapidly increasing, however, limited medications are presently available in the market (Birari and Bhutani, 2007). Obesity is a dyslipidemic disorder, wherein, derangement in lipid metabolism has been seen along with abnormal lipid levels (Bays et al., 2013), often associated with higher storage of lipid in adipocytes (Arner et al., 2011). It is interesting to note that dyslipidemia is associated with a cluster of diseases thereby, being a central player in development of metabolic syndrome (Jung and Choi, 2014). The manifestation of syndrome has decreased life expectancy and its quality (Katz et al., 2000; Pimenta et al., 2015; Taylor et al., 2013). Several nutritional theories have implicated diet as an important contributor in development of abnormal lipid levels (Kamran et al., 2016). It is stated that over nutrition with low metabolic output is major cause of developing dyslipidemia (Grundy and Barnett, 2004). In this context, cafeteria based diet which is enriched with high refined sugar and high fat serves as a powerhouse of excessive calories and when used in animal

Corresponding author: Dr. Laxmipriya Nampoothiri Associate Professor, Department of Biochemistry, Faculty of Science, The Maharaja Sayajirao University of Baroda,Vadodara-390002, Gujarat, India

E-mail: Ipnmsubaroda@gmail.com; Inampoothiri@gmail.com Tel.: +91-2652795594 models mimics all features of metabolic syndrome (Gomez-Smith et al., 2016; Sampey et al., 2011). Data from several reports also indicate that people increase the intake of high energy snack foods when stressed, thereby leading to dyslipidemic obesity (Anderson et al., 2011; Shori et al., 2017). This abnormal lipid status has affected immensely other metabolic pathways leading to cardiovascular risk. This has led to urgent need for developing the new antiobesity drugs that could manage dyslipidemia with fewer side effects. Currently, potential use of nutraceuticals for the management of dyslipidemia is not fully explored and could be an outstanding substitute approach for developing safe and effective dyslipidemic drugs. India, especially north-eastern region is a rich source of various medicinal plants. In this context, several herbs have been explored, amongst which Garcinia is of utmost importance due to its several important biological properties. Plants from the genus Garcinia have been reported from Asia, Africa and Polynesia. Anti-inflammatory, antinociceptive, antioxidant, antitumoral, antifungal, anticancer, antihistaminic, antiulcerogenic, antimicrobial, antiviral, vasodilatory, hypolipidemic, hepatoprotective, nephroprotec-tive and cardioprotective properties of plants from this genus have been reported (Santo et al., 2020). G. indica commonly known as "Kokum" is one of the Garcinia species used in traditional medicine in Asian countries as folk medicine to treat various ailments. In Ayurveda, it is known as Vrikshamla. Juice obtained from Kokum fruit or extracts prepared from aril or rind is used in the preparation of drugs in 
Indian, Chinese, Thai and Malaysian systems of medicine. Kokum finds its place in Ayurvedic systems of medicine and is considered to be beneficial for health (Swamy et al., 2014). Also, infusion prepared from the Kokum is used to treat piles, dysentery and infections. Furthermore, Kokum is known to strengthen the cardiovascular system and stabilize liver function (Braganza et al., 2012). Prophylactic potential of $G$. indica fruits for ailments as varied as rheumatism, rickets, enlargement of spleen, uterine complaints and in animal disorders have been described in Ayurveda. Its fruit juice or syrup is used as a coolant and helps to reduce body weight (Braganza et al., 2012). It is also used for getting relief in stomach and liver disorders (Bhat et al., 2005; Krishnamurthy, 1984; Krishnamurthy et al., 1982; Mishra et al., 2006). The present investigation was undertaken to investigate the efficacy of fruit juice of G. indica as a hypolipidemic agent in cafeteria based diet dyslipidemic rodent model.

\section{Material and Methods}

\subsection{Chemicals}

Hydroxycitric acid (HCA), calcium salt was purchased from Natural Remedies, Bengaluru, India. HPLC grade solvents (acetonitrile, trifluroacetic acid, TFA, and water) were purchased from Merck, Mumbai, India. Cholesterol (HiMedia Laboratories) and Orlistat (German Remedies) were purchased locally. Metabolic profile involving oral glucose tolerance test, lipid profile were performed using GOD-POD kit and lipid profile test kit was purchased from Enzopak (Rankem). Trizol, cDNA kit was procured from Takara Inc (PrimeScript 1st strand cDNA Synthesis Kit). Primers for metabolic enzymes were designed by primer express and synthesized by integrated DNA technologies. Serum hormones-insulin and leptin were assayed using ELISA kits (DBC Canada).

\subsection{G. indica fruit juice}

Mature fruits of G. indica were collected. Fruit pulp was compressed to get the juice. Collected juice was stored in glass bottles under refrigerated conditions till further use.

\subsection{Apparatus and chromatographic conditions for profiling of HCA in $G$. indica fruit juice}

Quantification of hydroxycitric acid was carried out using HPLC (Waters, USA) system consisting of quaternary pumps, an in-line vacuum degasser, and a photodiode array detector (PDA). The instrumentation was controlled by using Empower 3.0 software (Waters). The chromatographic separation was achieved using Sunfire ${ }^{\mathrm{TM}}$ C18 Column (4.6 x $250 \mathrm{~mm}, 5 \mu \mathrm{m}$, Waters, Milford, MA, USA) at ambient temperature. The mobile phases consisted of a mixture of solvents: $0.1 \%$ trifluoroacetic acid in water (A) and 0.1 $\%$ trifluoroacetic acid in acetonitrile (B). The optimised HPLC condition for gradient elution mode is as follows: elution was initially started with $90 \%$ of solvent (A) and $10 \%$ solvent (B) with a flow rate of $0.8 \mathrm{ml} / \mathrm{min}$. Further, after 10 min solvent (A) was decreased to $80 \%$ and solvent (B) was increased to $20 \%$. After $25 \mathrm{~min}$ of solvent (A) was decreased to $70 \%$ and solvent (B) was increased to $30 \%$. Solvent (A) was gradually decreased to $60 \%$ and solvent (B) was increased to $40 \%$ after $30 \mathrm{~min}$. At $31 \mathrm{~min}$ composition of solvent (A) and solvent (B) was brought back to $90 \%$ and $10 \%$, respectively. The flow rate through out the run was $0.8 \mathrm{ml} / \mathrm{min}$ and run time was $35 \mathrm{~min}$ (Kureshi et al., 2018). Detection wavelength was $211 \mathrm{~nm}$. Injection volume was $20 \mu \mathrm{l}$ (Kureshi et al., 2018).

\subsection{Animals}

Forty adult virgin female Charles Foster rats (3-4 months) showing regular estrous cyclicity were chosen for the study. Animals were housed in a standard controlled animal care facility $\left(22-25^{\circ} \mathrm{C}\right.$ and $45 \%$ humidity) in cages (one rats/cage) under equal dark and light cycle (1:1). Standard nutritional and environmental conditions for animals were maintained throughout the experiment (Chidrawar $e t$ al., 2011). All the experiments were carried out between 9:00 and 16:00 hours, at ambient temperature. The Nations Control and Supervision of Experiments on Animals (CPCSEA) guidelines were strictly followed and all the studies were approved by the Institutional Animal Ethical Committee (IAEC) (Protocol Number: BC/11/2017).

\subsection{Induction of dyslipidemia through diet}

The cafeteria diet was as described by Chidrawar et al. (2012). It consisted of 3 different diets: diet 1-condensed milk $(8 \mathrm{~g})+$ bread $(8$ $\mathrm{g})$; diet 2-chocolate $(3 \mathrm{~g})+$ biscuits $(6 \mathrm{~g})+$ dried coconut $(6 \mathrm{~g})$; diet 3 cheese $(8 \mathrm{~g})+$ boiled potatoes $(10 \mathrm{~g})$ for each animal. The three cafeteria diets were fed to each rat of the group that had 6 animals on days one, two, and three, respectively with repetition in the same succession for 10 weeks along with standard pellet diet. Cholesterol rich high fat diet (Kumar et al., 2008) was given to each rat four weeks prior to the $G$. indica fruit juice treatment.

\subsection{Treatment with $G$. indica fruit juice}

Animals were divided into six major groups, Group I (C) had animals that received lab chow diet and were considered as control. Further, animals fed with cafeteria diet and high-fat diets were divided into five groups, Group II (CD) consisted of animals which were fed with cafeteria diet and were considered to be dyslipidemic animals; Group III $(\mathrm{CD}+\mathrm{O})$ consisted orlistat (standard antiobesity drug) treated animals fed with cafeteria diet; Group IV $(\mathrm{CD}+\mathrm{G})$ were $G$. indica fruit juice treated animals fed with cafeteria diet; Group V(HF) animals were high fat diet treated animals, which served as positive control for dyslipidemia and Group VI $(\mathrm{HF}+\mathrm{G})$ were high-fat diet fed animals, which received $G$. indica fruit juice treatment. All G. indica fruit juice treatment was given daily orally for 30 days at a dose of $1 \mathrm{ml}$.

\subsection{Parameters analysed}

During 30 days of $G$. indica fruit juice treatment, all animals were continuously monitored for body weight and food intake. Oral glucose tolerance test (OGTT) was performed after $12 \mathrm{~h}$ fasting in all rats (Buchanan et al., 1991). Glucose (2g/kg body weight) was orally fed to the rats and blood samples was collected in sodium fluoride $(\mathrm{NaF})$ and EDTA coated tubes considered as 0 minute. After that blood sample was collected at different time intervals $(30,60,90$, and 120 minutes) and plasma was used for the estimation of glucose. Analysis of the lipid profile was done by Enzopak kits which estimated total cholesterol, triglycerides, HDL-C, and LDL-C from serum. Serum hormones like insulin and leptin were assayed by ELISA method. Homeostatic model assessment, Insulin Resistance (HOMA-IR) was calculated based on the fasting blood glucose and fasting insulin values using the following formula HOMA IR $=$ (Fasting insulin $\mathrm{x}$ Fasting glucose)/405. Normal insulin resistance: $<3$; moderate insulin resistance: between 3-5; severe insulin resistance: $>5$. In addition to 
this, 3-hydroxy-3-methyl-glutaryl-coenzyme A reductase (HMG-CoA reductase) activity was carried out according to the protocol described by (Rao and Ramakrishnan, 1975). Also, plasma lecithin cholesterol acyl transferase (LCAT) activity was assayed by the method described by Hitz (Hitz et al., 1983). In addition to this, gene expression of cholesterol biosynthetic enzymes like Acetyl-CoA carboxylase (ACC) was evaluated by RT-PCR. RNA was isolated using trizol reagent following which 2 microgram of RNA was subjected to cDNA preparation. cDNA was later used for expression studies. Primer details are indicated in Table 1. Serum glutamate pyruvate transaminase (SGPT) and creatinine were analyzed to understand the toxicity of $G$. indica fruit juice.

Table 1: Sequences of target gene specific primers

\begin{tabular}{|l|l|l|}
\hline Gene name & Accession number & Sequence of primers \\
\hline Acetyl-coenzyme A carboxylase & NM_022193.1 & F-5' ATGGTCTACATTCCCCCACA 3' \\
\cline { 2 - 3 } & & R-5' ATCACAACCCAAGAACCACC 3' \\
\hline GAPDH (internal control) & NM_017008 & F-5'CAAGGTCATCCATGACAACTTTG 3' \\
\cline { 2 - 3 } & & R-5' GTCCACCACCCTGTTGCTGTAG 3' \\
\hline
\end{tabular}

\subsection{Statistical analysis}

Comparison of different groups was done using analysis of variance (ANOVA) and Student $t$-test. The analysis was carried out using GraphPad version 5.0. $P \geq 0.05$ was considered significant. All results are expressed as the mean \pm SEM for 6-7 animals in each group.

\section{Results}

\subsection{HPLC analysis of $G$ indica fruit juice}

Identification and quantification of the HCA in G. indica fruit juice was carried out with the comparison of retention time and PDA spectra of the peak of standard HCA (Figure 1A). Peak of HCA eluted at 5.3 min. Furthermore, identity of peak of HCA was also confirmed by spiking studies. Quantification of HCA was carried out using a calibration curve prepared from the different concentration of standard HCA and the content of (-)-hydroxycitric acid (HCA) in G. indica juice was $16.57 \%$ (Figure 1B).
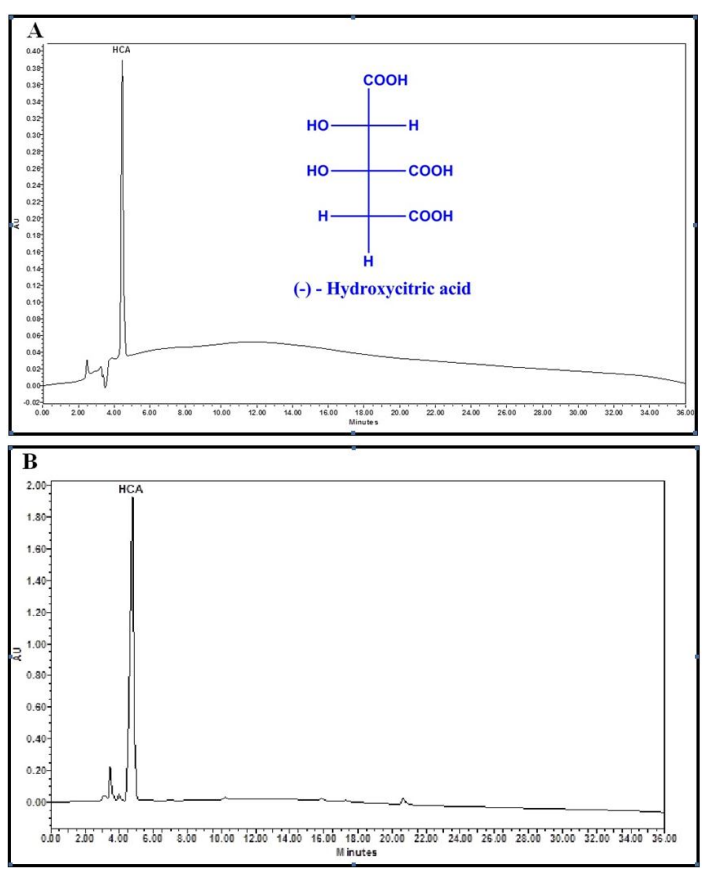

Figure 1: HPLC analysis of $G$. indica fruit juice. A peak of standard HCA B. Quantification of HCA in G. indica fruit juice.
3.2 Effect of $G$. indica fruit juice on body weight, food and water intake

Figure 2A. represents the effect of $G$. indica fruit juice on the food consumption of the animals. There was significant increase in body weight observed after 10 weeks of treatment in cafeteria group as compared to control group as shown in Figure 2B. After 4 weeks of treatment with $G$. indica fruit juice, it was observed that there was significant decrease in body weight as compared to non-treated cafeteria group as well as orlistat treated cafeteria group. Thereby, treatment of $G$. indica fruit juice for 4 weeks was effective in reduction of body weight in the animals.

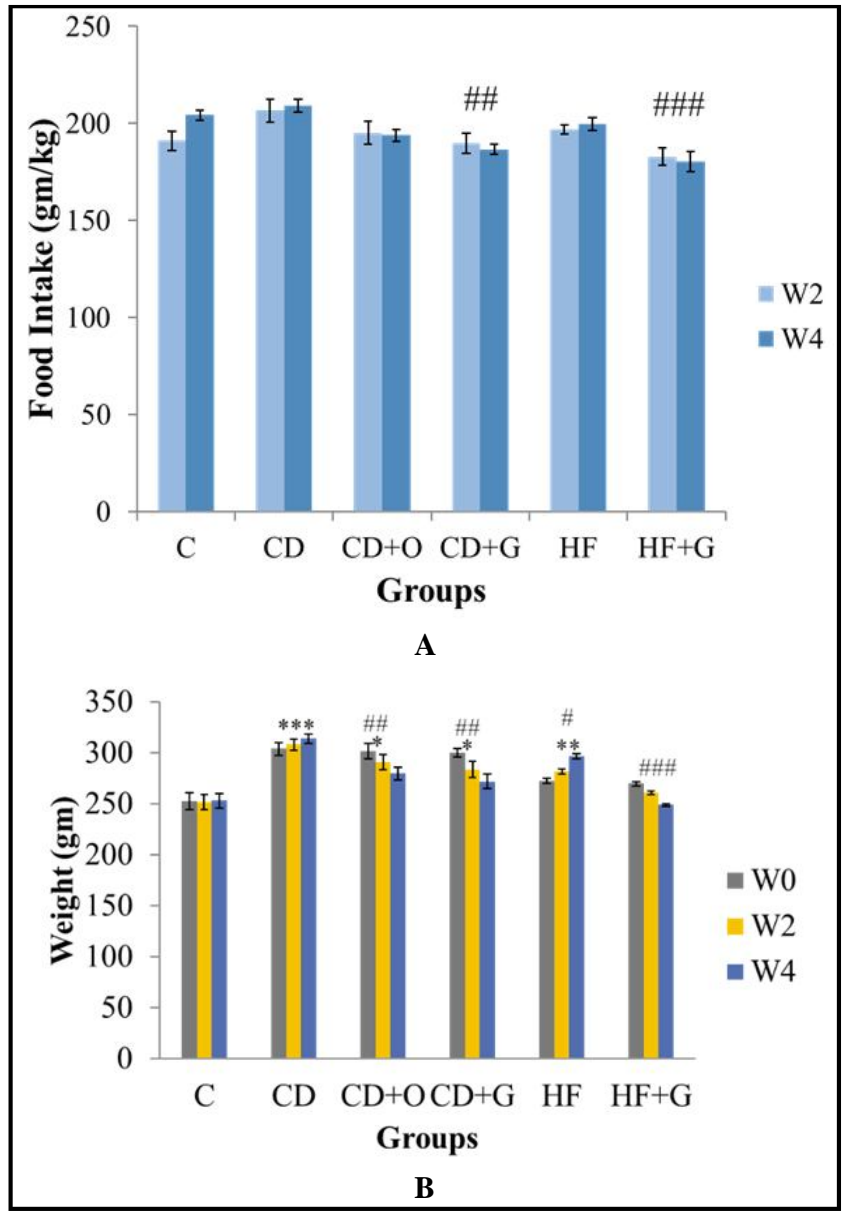




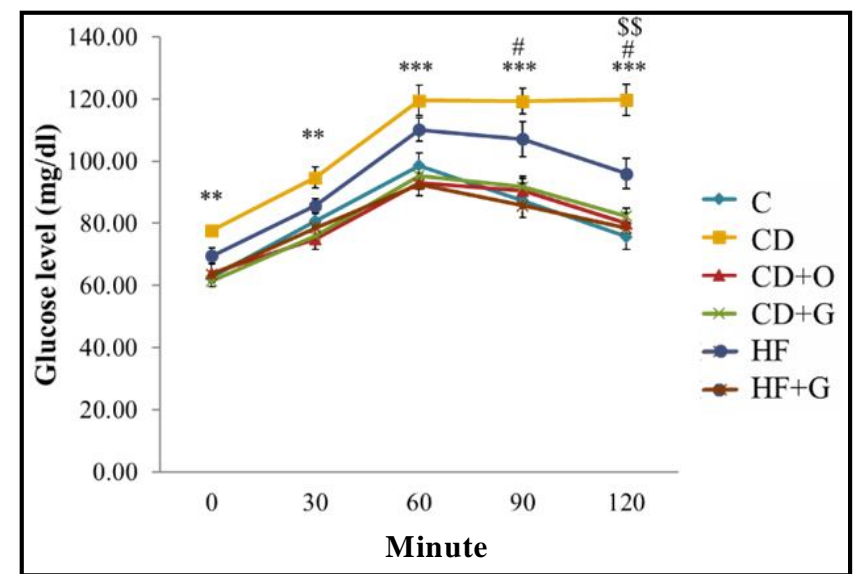

Figure 2: Effect of $G$ indica fruit juice on A. food intake, B. body weight. * comparison with control, \# comparison with cafeteria diet, $\mathrm{N}=5$, values are Mean $\pm \mathrm{SEM}$, \# $p<0.05$, \#\# $p<0.01, \# \# \#<<0.001, * p<0.05, * * p<0.01$, $* * * p<0.001, \mathrm{~W} 0, \mathrm{~W} 2, \mathrm{~W} 4$ represents week 0 (before treatment), week 2 and week 4 (after treatment) $C$. glucose tolerance. * comparison with cafeteria diet, \# comparison with high-fat diet, \$ comparison between cafeteria diet and high-fat diet $\mathrm{N}=5$, values are Mean \pm SEM, \# $p<0.05, * * p<0.01, * * * p<0.001$, $\$ \$ p<0.01$.

\subsection{Effect of $G$ indica fruit juice on metabolic parameters}

High fat affects insulin sensitivity. Thereby, oral glucose tolerance test was performed after $G$. indica fruit juice treatment. Figure 2C demonstrated that the treatment with G. indica fruit juice for 4 weeks significant decrease in glucose intolerance was recorded. This data was comparable to group treated with orlistat, thereby, suggesting that $G$. indica fruit juice has potential to improve glucose sensitivity. However, cafeteria group and high fat group were glucose intolerant. In addition to this, serum hormone profile for insulin and leptin along with HOMO-IR demonstrated that there was significant increase in serum insulin level in cafeteria treated group (Table 2). Upon treatment with G. indica fruit juice, there was significant decrease in serum insulin level. Similar results were observed in orlistat treated group. In case of high-fat group, no significant change was observed as compared to control group. Serum leptin levels were significantly very high as compared to control group in cafeteria treated group. $G$. indica fruit juice treatment showed significant decrease in its level. While calculating HOMO-IR, it was observed, there is severe insulin resistance in cafeteria treated group. After treatment with $G$. indica fruit juice and orlistat, its value decreased but still it was showing moderate insulin resistance similar to control group. Thereby, it could be possible that long term treatment may improve glucose sensitivity.

Table 2: Effect of $G$. indica fruit juice on hormone profile

\begin{tabular}{|l|l|l|c|}
\hline Groups & Insulin $(\mathbf{m I U} / \mathbf{m l})$ & Leptin $(\mathbf{n g} / \mathbf{m l})$ & HOMO-IR \\
\hline $\mathrm{C}$ & 24.09 & 0.74 & 3.73 \\
\hline $\mathrm{CD}$ & $36.80^{* *}$ & $1.17 * * *$ & 7.06 \\
\hline $\mathrm{CD}+\mathrm{O}$ & $32.10 \#$ & $0.84 \# \# \#$ & 5.07 \\
\hline $\mathrm{CD}+\mathrm{G}$ & $28.47 \# \#$ & $0.76 \# \# \#$ & 4.31 \\
\hline $\mathrm{HF}$ & $29.37 * \# \#$ & $0.98 * * \# \#$ & 5.04 \\
\hline $\mathrm{HF}+\mathrm{G}$ & 27.36 & 0.81 & 4.29 \\
\hline
\end{tabular}

* Comparison with Control, \# Comparison with cafeteria diet. $\mathrm{n}=5$, Values are represented as Mean $\pm \mathrm{SEM}$, \# $p<0.05$, \#\# $p<0.01$, \#\#\# $p<0.001 * p<0.05, * * \quad<<0.01, * * * \quad p<0.001$.

Table 3 represents lipid profile after administration of G. indica fruit juice for 30 days. There was significant decrease in serum total cholesterol, serum tryglycerides and LDL-cholsterol and increase in HDL-cholesterol. It suggested that dyslipidemic rat model was reverting back to normal phenotype. These data when compared to standard drug shows similar result. Cafeteria treated group and highfat treated group both shows significant alteration in lipid profile.

Table 3: Effect of $G$. indica fruit juice on lipid profile.

\begin{tabular}{|l|l|l|l|l|}
\hline Groups & Total cholesterol $(\mathbf{m g} / \mathbf{d l})$ & HDL-cholesterol $(\mathbf{m g} / \mathbf{d l})$ & Triglycerides $(\mathbf{m g} / \mathbf{d l})$ & LDL-cholesterol $(\mathbf{m g} / \mathbf{d l})$ \\
\hline $\mathrm{C}$ & $55.55 \pm 3.54$ & $32.94 \pm 1.09$ & $80.83 \pm 4.05$ & $6.445 \pm 4.63$ \\
\hline $\mathrm{CD}$ & $95.12 \pm 2.90 * * *$ & $22.06 \pm 0.86 * *$ & $141.9 \pm 6.36 * * *$ & $44.69 \pm 4.03 * * *$ \\
\hline $\mathrm{CD}+\mathrm{O}$ & $78.65 \pm 3.75 \#$ & $32.16 \pm 0.57 \# \#$ & $97.98 \pm 6.64 \# \#$ & $26.9 \pm 4.47 \# \#$ \\
\hline $\mathrm{CD}+\mathrm{G}$ & $80.32 \pm 1.58 \#$ & $31.3 \pm 0.43 \# \#$ & $95.3 \pm 2.33 \# \#$ & $29.96 \pm 1.37 \# \#$ \\
\hline $\mathrm{HF}$ & $86.16 \pm 2.10 * * *$ & $28.72 \pm 0.78 *$ & $118.1 \pm 7.61 * *$ & $33.83 \pm 1.88 * *$ \\
\hline $\mathrm{HF}+\mathrm{G}$ & $82.92 \pm 2.37$ & $30.29 \pm 0.96$ & $107.5 \pm 6.90$ & $31.14 \pm 1.23$ \\
\hline
\end{tabular}

* Comparison with Control, \# Comparison with cafeteria diet $\mathrm{n}=5$, Values are represented as Mean $\pm \mathrm{SEM}$, ** $p<0.01, * * * p<0.001, \# p<0.05$, \#\# $p<0.01$.

3.4 Effect of G. indica fruit juice on lipid metabolizing enzymes

Figure $3 \mathrm{~A}$ represents the activity of key lipid metabolizing enzyme HMG-CoA reductase. Upon treatment of $G$. indica fruit juice, HMGCoA reductase activity was decreased, thus indicated a decreased cholesterol biosynthesis. Ideal decrease in activity was seen on treatment with orlistat. High-fat group does not show significant change suggesting no change, in HMG-Co reductase activity. Figure 3B shows the effect of $G$. indica fruit juice on LCAT activity. On administering G. indica fruit juice, a significant increase in LCAT activity was observed as compared to the cafeteria group. This activity was not increased in case of orlistat treated group. While high-fat treated groups showed decrease in LCAT activity. No significant change was observed in G. indica fruit juice treated group as compared to high-fat treated group. Figure $3 \mathrm{C}$ represents transcript level of enzyme acetyl Co-a carboxylase, a lipid metabolizing enzyme. As seen in the figure, cafeteria diet treated group shows significant increase in ACC level as compared to control. 


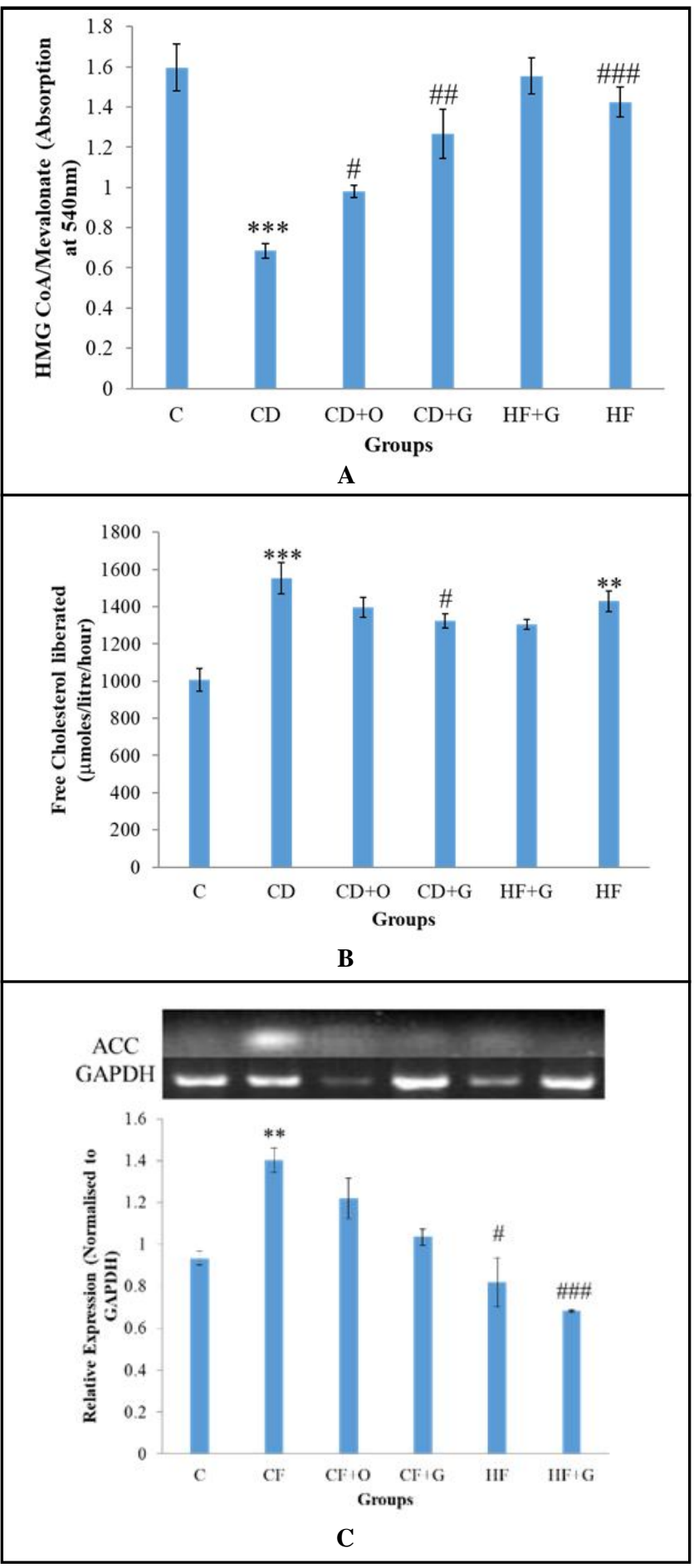

Figure 3: Effect of $G$. indica fruit juice on lipid metabolizing enzymes A. HMG CoA reductase activity B. LCAT activity and C. gene expression level of ACC enzyme. * comparison with control, \# comparison with cafeteria diet, $N=5$, Values are Mean $\pm \mathrm{SEM}$, \# $<0.05$, $\# p<0.01, \# \# p<0.001, * * p<0.01, * * * p<0.001, \mathrm{C}$. relative gene expression of ACC.* comparison with control, \# comparison with cafeteria diet. $n=5$, values are Mean \pm SEM, \# $p<0.05$, \#\#\# $p<0.001, * *<0.01$.
After treatment of $G$. indica fruit juice for 30 days, there was marked decrease in its level. The data is similar to the orlistat treated group. High-fat diet treated group does not show significant change in ACC level as compared to control group.

\subsection{Effect of G. indica fruit juice on toxicity parameters}

Figure 4 describes the toxicity parameters of liver and kidney upon administration with the G. indica fruit juice. As seen in Figure 4A, there was significant increase in SGPT activity, suggesting liver toxicity due to cafeteria diet treatment as compared to control. Upon the administration of $G$. indica fruit juice, there was significant decrease in its activity. However, there was no significant change in high-fat treated group. Figure 4B represents serum creatinine level and as seen in the Figure, there was significant increase in serum creatinine level in cafeteria treated group and the level decreased in $G$. indica fruit juice treated group. In case of high-fat treated group, significant increase in serum creatinine level was recorded.
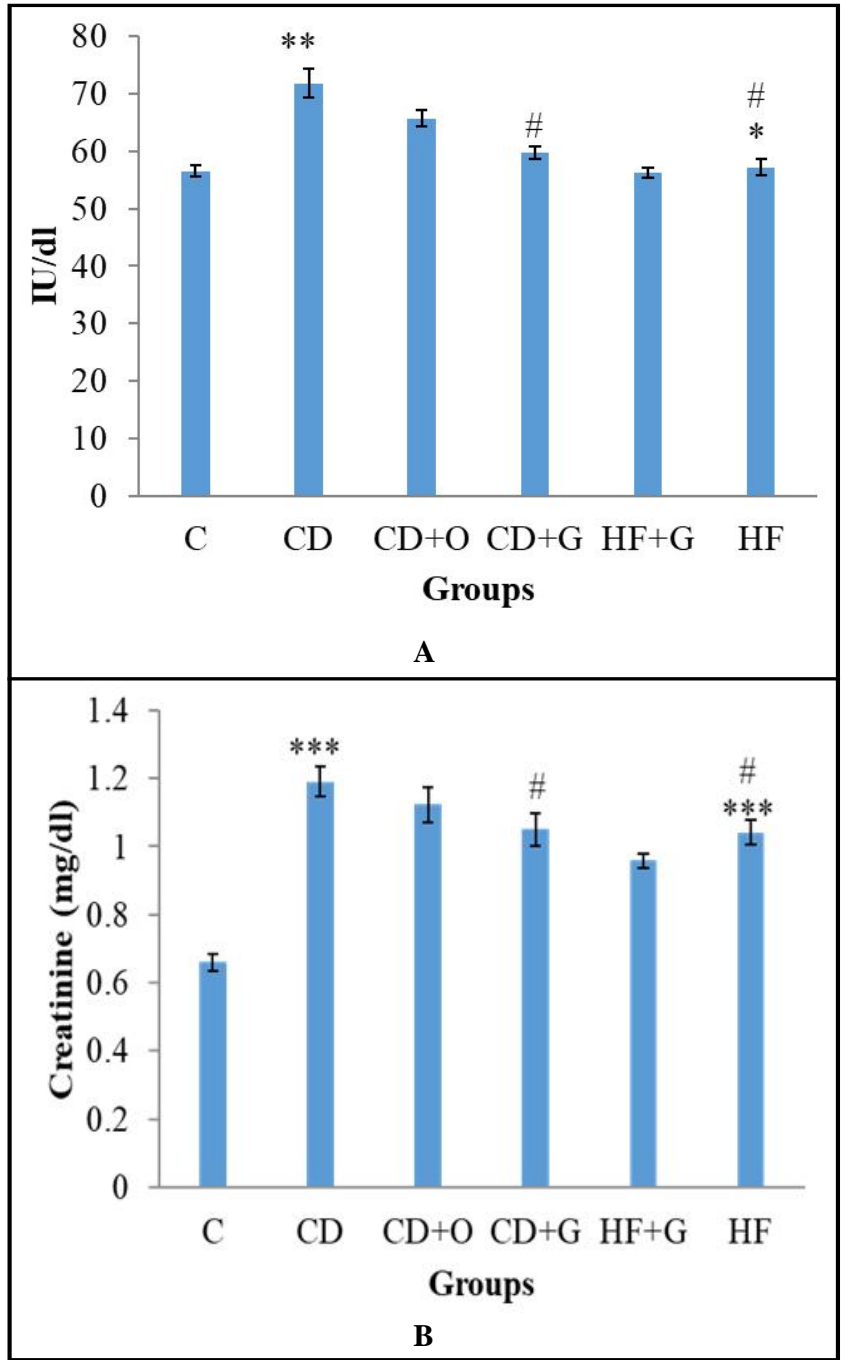

Figure 4: Effect of $G$. indica fruit juice on toxicity parameters of A. liver by SGPT activity and B. kidney by creatinine level. * comparison with control, \# comparison with cafeteria diet. $\mathrm{N}=5$, values are Mean $\pm \mathrm{SEM}, * * p<0.01, * * * p<0.001, \# p<0.05$. 


\section{Discussion}

Obesity is a one of major dyslipidemia associated metabolic syndrome. Dyslipidemia is one of the initial steps for the development of obesity. In present study, cafeteria diet was used to generate a metabolic syndrome model for dyslipidemia to study initial stages for obesity. During dyslipidemia, there is increase in glucose intolerance, which is one of the important factor of metabolic syndrome and this may be due to increase in free fatty acid (Nambi et al., 2002). OGTT profile was observed upon cafeteria diet administration, suggesting that enriched fat diet for 10 weeks is sufficient to cause dyslipidemia. In dyslipidemia, lipoprotein metabolism is altered which results in either overproduction or deficiency in certain lipoprotein molecule ( Meisinger et al., 2006). There may be alteration in the levels of either one or more lipoprotein molecules followed by elevated levels of total cholesterol, low density lipoprotein and triglycerides along with decreased levels of high density lipoprotein which later on can be developed in obesity (Mishra et al., 2005; Misra et al., 2006; Snehalatha, 2003; Vikram et al., 2003). In our model, it was observed that there was significant increase in total cholesterol, LDL-cholesterol and triglycerides. Also, significant decrease in HDL-cholesterol was also observed. These all parameters are major characteristics of dyslipidemia (Nambi et al., 2002).

HMG-CoA-Reductase catalyses the rate limiting step of liver cholesterol biosynthesis ( Bucher et al., 1960; Siperstein,. 1966). In dyslipidemia, it was observed that HMG-CoA-reductase activity was higher as compared to control group (Wu et al., 2013). In our study, it was observed that there was significant decrease in substrate to product ratio suggesting an increase in HMG-CoA-reductase activity. Increase in its activity has resulted in increase in serum cholesterol levels after 10 weeks of treatment. LCAT is an enzyme that catalyses transfer of fatty acids from phosphatidylcholine to the hydroxyl residue (Assmann et al., 1978). Acetylation of cholesterol is helpful for clearance of excess cholesterol. LCAT promotes maturation of HDL particles in plasma and transport cholesterol maintaining a concentration gradient for the diffusion of cellular un-esterified cholesterol to HDL-cholesterol (Shigematsu et al., 2001). In diet induced obese rats, it was observed that there was significant decrease in LCAT activity which correlates with decrease in HDL-cholesterol (Subash and Augustine, 2014). Current study demonstrates that in dyslipidemia, rats there was significant decrease in LCAT activity which can be correlated with low HDL-cholesterol.

Dyslipidemia is a major symptom in development of metabolic syndrome (Mishra et al., 2007). There are several medications available for dyslipidemia. Two major classes are statins and fibrtaes (Wierzbicki et al., 2003). But, due to high cost and hazardous sideeffects, there is need for new and better therapy in management of dyslipidemia. Plant derived products or nutraceuticals can be potential targets for the development of new drugs (Chidrawar et al., 2012), as it renders few side-effects. In this context, several plants have been explored for the hypolipidemic effects like Aloe barbadensis Mill., Enicostemma littorale, G. cambogia (Desai et al., 2012; Oluyemi et al., 2002; Vasu et al., 2005). G. indica is an indigenous plant however, medicinal properties of this plant are not well studied. Thereby, our present study, involves evaluating efficacy of $G$. indica for its hypolipidemic property.
After the treatment of $G$. indica fruit juice, there was significant decrease in body weight as compared to non-treated groups, suggesting that the G. indica fruit juice could be used for the development of anti-dyslipidemic drugs. Similar result was observed in the rats treated with standard drug. Similarly glucose intolerance was reduced in the G. indica fruit juice treated group as compared to non-treated group. In case of positive control (high-fat) group, no significant change in the body weight was observed as compared to control group, suggesting that dyslipidemic rat model was not developed. Glucose intolerance is a characteristic feature of insulin resistance, therefore, serum insulin levels were measured (Vikram et al., 2003). There was significant increase in serum insulin level in cafeteria group as compared to control group. After the treatment with $G$. indica fruit juice, there was significant reduction in insulin level. It can be correlated with serum triglycerides level. HOMO-IR is used for evaluation of insulin resistance. While calculating HOMOIR, it was found that the animals treated with cafeteria diet showed significant increase in index suggesting severe insulin resistance. While treatment with G. indica fruit juice resulted in decrease in index but the values were still in the range of moderate insulin resistance. During dyslipidemic obesity, there is increased level of circulating leptin level (Dobrian et al., 2000). Serum leptin levels were elevated in cafeteria treated and high-fat diet treated groups as compared to control. After treatment with G. indica fruit juice, it was observed that there was significant reduction in serum leptin levels.

Lipid profile showed significant change in the G. indica fruit juice treated groups as compared to non-treated ones. Serum total cholesterol, triglycerides and LDL-cholesterol were back to normal in G. indica fruit juice treated group as compared non-treated group. It suggests that $G$. indica fruit juice is altering the lipid metabolism pathway resulting in normal phenotype.

HMG-CoA-reductase and LCAT activity were measured to check effect of G. indica fruit juice on lipid metabolising enzymes. After treatment, it was observed that in there was significant increase in the ratio of substrate to product resulting in decreased HMG-CoAreductase activity. This might be the reason for decreased cholesterol level. Group treated with standard drug also showed the similar result, but to a lower extent. LCAT activity was significantly increased in $G$. indica fruit juice treated group as compared to non-treated group. In case of group treated with standard drug, no significant change was observed. mRNA transcripts levels of acetyl-coenzyme A carboxylase (ACC) were also checked. ACC is an enzyme responsible for the production and regulation of fatty acid. It is predominately expressed in the liver and it is inducible in response to feeding. Transcript level of ACC is increased in obesity resulting in production of free fatty acid (Jeffery et al., 2013). mRNA levels of ACC were increased in cafeteria treated group. As a result of administration of G. indica fruit juice, significant reduction in its level was observed, suggesting decrease in free fatty acid levels. However, other transcriptional factors need to be evaluated for further confirmation. Thereby, present study has clearly shown the G. indica fruit juice could be a strong candidate for a hypolipidemic drug. Further, investigations needs to be done to mark the product as a dyslipidemic nutraceutical. Liver and kidney function tests showed that after 10 weeks of treatment with cafeteria diet there was significant increase in SGPT activity and serum creatinine, respectively, suggesting that prolong dose of cafeteria diet can alter liver and kidney function (Chidrawar et al., 2012). After treatment with $G$. indica fruit juice, it was observed that there was significant 
decrease in SGPT activity and serum creatinine levels. It suggests that G. indica fruit juice may have potential to restore liver and kidney functions. These results were not observed in the group treated with standard drug.

\section{Conclusion}

Increasing evidences are suggesting the significant impact of plant derived phytocomponents in the regulation of different aspects of human physiology and thereby, aid in the treatment and management of cardiovascular diseases and metabolic syndrome like obesity and dyslipidemia. In the current study, cafeteria diet induced obesity model was successfully developed which exhibited the associated complications of metabolic syndrome such as increased body weight, dyslipidemia, and insulin resistance after 10 weeks. Also, the potential of G. indica fruit juice to ameliorate dyslipidemia was evaluated in cafeteria diet fed obese rat model. It was observed that treatment with $G$. indica fruit juice at a dose of $1 \mathrm{ml}$ for 4 weeks could restore the body weight, dyslipidemia, glucose sensitivity, insulin resistance and expression and activities of key lipid metabolizing enzymes in cafeteria diet induced dyslipidemic rat model. The observed therapeutic potential was better than orlistat, which is the standard drug used for treatment of dyslipidemia and obesity. Thereby, suggesting that phytocomponents such as HCA present in G. indica fruit juice is a potent anti-dyslipidemic agent without inducing toxicity. However, phytocompounds from G. indica can be further analysed for understanding its molecular targets towards amelioration of dyslipidemia.

\section{Acknowledgements}

The authors acknowledge Institutional Animal Ethical committee (IAEC) members, Department of Biochemistry for the animal ethical clearance. One of the authors, Azazahemad A. Kureshi (Senior Research Fellow, UGC-MANF) (Award Number-201516-MANF2015-17-GUJ-49309) gratefully acknowledges the University Grants Commission (UGC) and Ministry of Minority Affairs, New Delhi, Govt. of India for providing financial assistance. Authors (SK and RS) acknowledge ICAR, New Delhi for funding a research project "Network Project on High Value Compounds/Phytochemicals".

\section{Conflict of interest}

The authors declare that there are no conflicts of interest relevant to this article.

\section{References}

Anderson, B.; Rafferty, A.Ps.; Lyon-Callo, S.; Fussman, C. and Imes, G. (2011). Fast-food consumption and obesity among Michigan adults. Prev. Chronic Dis., 8:A71.

Arner, P.; Bernard, S.; Salehpour, M.; Possnert, G.; Liebl, J.; Steier, P.; Buchholz, B.A.; Eriksson, M.; Arner, E.; Hauner, H.; Skurk, T.; Rydén, M.; Frayn, K.N. and Spalding, K.L. (2011). Dynamics of human adipose lipid turnover in health and metabolic disease. Nature, 478:110-113. https://doi.org/ $10.1038 /$ nature 10426 .

Assmann, G.; Schmitz, G. and Heckers, H. (1978). The role of high density lipoproteins in lecithin: Cholesterol acyltransferase activity: Perspectives from Tangier disease. Scand. J. Clin. Lab. Invest., 38(sup150):98-102. https://doi.org/10.1080/00365517809104907
Bays, H.E.; Toth, P.P.; Kris-Etherton, P.M.; Abate, N.; Aronne, L.J.; Brown, W.V.; Gonzalez-Campoy, J.M.; Jones, S.R.; Kumar, R.; La Forge; R. and Samuel, V.T. (2013). Obesity, adiposity, and dyslipidemia: A consensus statement from the National Lipid Association. J. Clin. Lipidol., 7(4):304383. https://doi.org/10.1016/j.jacl.2013.04.001

Bhat, D.J.; Kamat, N. and Shirodkar, A. (2005). Compendium and Procedings of $2^{\text {nd }}$ National Seminar on Kokum (Garcinia indica Choicy), Goa University, Goa.

Birari, R.B. and Bhutani, K.K. (2007). Pancreatic lipase inhibitors from natural sources: Unexplored potential. Drug Discov. Today, 12(1920):879-889. https://doi.org/10.1016/j.drudis.2007.07.024

Braganza, M.; Shirodkar, A.; Bhat, D.J. and Krishnan, S. (2012). Resource Book on KOKUM (Garcinia indica Choisy), Western Ghats Kokum Foundation, Panaji, Goa, India.

Buchanan, T.A.; Sipos, G.F.; Gadalah, S.; Yip, K.P.; Marsh, D.J.; Hsueh, W. and Bergman, R.N., (1991). Glucose tolerance and insulin action in rats with renovascular hypertension. Hypertension, 18(3):341-347.

Bucher, N.L.R.; Overath, P. and Lynen, F. (1960). $\beta$-hydroxy- $\beta$-methylglutaryl coenzyme a reductase, cleavage and condensing enzymes in relation to cholesterol formation in rat liver. BBA - Biochim. Biophys. Acta, 40:491-501. https://doi.org/10.1016/0006-3002(60)91390-1

Chidrawar, V. R; Patel, K.N.; Shiromwar, S.S. and Kshirsagar, A. D. (2011). Exploiting antiobesity mechanism of Clerodendrum phlomidis against two different models of rodents. Int. J. Green. Pharm., 5:216-228.

Chidrawar, V. R.; Patel, K.N., Bothra, S.; Shiromwar, S.S., Koli, A.R. and Kalyankar, G.G. (2012). Antiobesity effect of Stellaria media methanolic extract in the murine model of cafeteria diet induced obesity. Int. J. Nutr. Pharmacol. Neurol. Dis., 2(2):121. https://doi.org/10.4103/22310738.95963

Desai, B.N.; Maharjan, R.H. and Nampoothiri, L.P. (2012). Aloe barbadensis Mill. formulation restores lipid profile to normal in a letrozoleinduced polycystic ovarian syndrome rat model. Pharmacognosy Res., 4:109-115. https://doi.org/10.4103/0974-8490.94736

Dobrian, A.D.; Davies, M.J.; Prewitt, R.L. and Lauterio, T.J. (2000). Development of hypertension in a rat model of diet-induced obesity. Hypertension, 35(4):1009-1015. https://doi.org/10.1161/01.HYP.35.4.1009.

Espirito Santo, B.L.S.; Santana, L.F.; Kato Junior, W.H.; de Araujo, F.O.; Bogo, D.; Freitas, K.C.; Guimaraes, R.C.A.; Hiane, P.A.; Pott, A.; Filiu, W.F.O.; Asato, M.A.; Figueiredo, P.O. and Bastos, P.R.H.O. (2020). Medicinal potential of Garcinia species and their compounds. Molecules, 25:4513 doi: 10.3390/molecules25194513.

Gomez-Smith, M.; Karthikeyan, S.; Jeffers, M.S.; Janik, R.; Thomason, L.A.; Stefanovic, B. and Corbett, D. (2016). A physiological characterization of the Cafeteria diet model of metabolic syndrome in the rat. Physiol. Behav., 167(1):382-391. https://doi.org/10.1016/j.phys beh.2016.09.029

Grundy, S.M. and Barnett, J.P. (2004). Metabolic and health complications of obesity. Disease-a-Month, 36(12):641-731. https://doi.org/ 10.1016/0011-5029(90)90015-j

Hitz, J.; Steinmetz, J. and Siest, G. (1983). Plasma lecithin: Cholesterol acyltransferase - reference values and effects of xenobiotics. Clin. Chim. Acta., 133(1):85-96. https://doi.org/10.1016/0009-8981(83) 90023-2.

Jung, U.J. and Choi, M.S., (2014). Obesity and its metabolic complications: The role of adipokines and the relationship between obesity, inflammation, insulin resistance, dyslipidemia and nonalcoholic fatty liver disease. Int. J. Mol. Sci. 15(4):6184-6223. https://doi.org/ 10.3390/ijms15046184. 
Kamran, A.; Sharifirad, G.; Heydari, H. and Sharifian, E. (2016). The effect of theory based nutritional education on fat intake, weight and blood lipids. Electron. Physician, 8:3333-3342. https://doi.org/10.19082/ 3333.

Katz, D.A.; McHorney, C.A. and Atkinson, R.L. (2000). Impact of obesity on health-related quality of life in patients with chronic illness. J. Gen. Intern. Med., 15:789-796. https://doi.org/10.1046/j.1525-1497. 2000.90906.x

Krishnamurthy, N. (1984). Chemical and technological studies on colouring matters from natural sources for use in foods. Ph.D Thesis, Mysore University.

Kumar, V.; Singh, S.; Khanna,A.S.; Khan, M; Chander, R.; Mahdi, F.; Saxena, J.K.; Singh, R. and Singh, R.K. (2008). Hypolipidemic activity of Anthocephalus indicus (kadam) in hyperlipidemic rats. Med. Chem. Res., 17(2-7):152-158.doi.org/10.1007/s00044-007-9045-4.

Kureshi, A.A.; Dholakiya, C.; Hussain, T.; Mirgal, A.; Salvi, S.P.; Barua, P.C.; Talukdar, M.; Beena, C.; Kar, A.; Zachariah, T.J.; Kumari P.; Dhanani, T.; Patel, J.; Singh, R. and Kumar, S. (2018). Comparative profiling of hydroxycitric acid in fruit juice of five Garcinia species from India. XXVIII Annual Conference of Indian Association for Angiosperm Taxonomy and International Symposium on Conservation of Angiosperm Diversity: Hidden Treasure of Today and Tomorrow, M S University, Vadodara, Gujarat, pp:137-138.

Meisinger, C.; Döring, A.; Thorand, B.; Heier, M. and Löwel, H. (2006). Body fat distribution and risk of type 2 diabetes in the general population: Are there differences between men and women? The MONICA/ KORA Augsburg Cohort Study. Am. J. Clin. Nutr., 84(3):483-489. https://doi.org/10.1093/ajen/84.3.483.

Mishra, A.; Bapat, M.M.; Tilak, J.C. and Devasagayam, T.P.A. (2006). Antioxidan activity of Garcinia indica (Kokam) and its syrup. Curr. Sci., 91(1), 90-93

Misra, A.; Misra, R.; Wijesuriya, M. and Banerjee, D. (2007). The metabolic syndrome in South Asians: Continuing escalation and possible solutions. Indian J. Med. Res., pp:345-354

Misra,A.; Vikram, N.K.; Gupta, R.; Pandey, R.M.; Wasir, J.S. and Gupta, V.P. (2006). Waist circumference cutoff points and action levels for Asian Indians for identification of abdominal obesity. Int. J. Obes., 30:106-111. https://doi.org/10.1038/sj.ijo.0803111.

Misra, A.; Wasir, J.S. and Pandey, R. (2005). An evaluation of candidate definitions of the metabolic syndrome in adult Asian Indians. Diabetes Care, 28(2):398-403. https://doi.org/10.2337/ diacare.28.2.398

Nambi, V.; Hoogwerf, B.J. and Sprecher, D.L. (2002). A truly deadly quartet: Obesity, hypertension, hypertriglyceridemia, and hyperinsulinemia Cleve. Clin. J. Med., 69(12):985-989. https://doi.org/10.3949/ ccjm.69.12.985.

Oluyemi, K.; Jimoh, O.R.; Adesanya, O.A.; Omotuyi, I.A.; Josiah, S.J. and Oyesola, T.O. (2007). Effects of crude ethanolic extract of Garcinia cambogia on the reproductive system of male wistar rats (Rattus novergicus). African J. Biotechnol., 6:1236-1238.

Pimenta, F.B.C.; Bertrand, E.; Mograbi, D.C.; Shinohara, H. and Landeira-Fernandez J. (2015). The relationship between obesity and quality of life in Brazilian adults. Front. Psychol., 6:1-7. https://doi.org/10.3389/ fpsyg.2015.00966
Rao, A.V. and Ramakrlshnan, S. (1975). Indirect assessment of Hydroxymethylglutaryl-CoA reductase (NADPH) activity in liver tissue. Clin. Biochem., 21:1523-1525.

Sampey, B.P.; Vanhoose, A.M.; Winfield, H.M.; Freemerman, A.J.; Muehlbauer, M.J.; Fueger, P.T.; Newgard, C.B. and Makowski, L. (2011). Cafeteria diet is a robust model of human metabolic syndrome with liver and adipose inflammation: Comparison to high-fat diet. Obesity, 19(6):11091117. https://doi.org/10.1038/oby.2011.18.

Shigematsu, N.; Asano, R.A.; Shimosaka, M.S. and Okazaki, M. (2001). Effect of administration with the extract of Gymnema sylvestre $\mathrm{R} . \mathrm{Br}$ on plasma and liver lipid in rats. Biol Pharm Bull., 24(6):713-717.

Shori, A.B.; Albaik, M. and Bokhari, F.M. (2017). Fast food consumption and increased body mass index as risk factors for weight gain and obesity in Saudi Arabia J. Obes. Med., 8:1-5. https://doi.org/10.1016/ j.obmed.2017.09.002

Siperstein, M. and Fagan, V. (1996). Feedback control of mevalonate synthesis by diettary cholesterol.The Journal of Biological Chemisrty, 241:602-609.

Snehalatha, C.; Viswanathan, V. and Ramachandran, A. (2003). Cutoff values for normal anthropometric variables in Asian Indian adults. Diabetes Care, 26(5):1380-1384. https://doi.org/10.2337/diacare.26.5.1380

Subash, A. and Augustine, A. (2014). Hypolipidemic effect of methanol fraction of Aconitum heterophyllum wall ex Royle and the mechanism of action in diet-induced obese rats. J. Adv. Pharm. Technol. Res., 3(4):224-228. https://doi.org/10.4103/22314040.104713

Swami, S.; Thakor, N.J. and Patil, S.C. (2014). Kokum (Garcinia indica) and its many functional components as related to the human health : A review. J. Food Res. Technol., 2:130-142.

Taylor, V.H.; Forhan, M.; Vigod, S.N.; McIntyre, R.S. and Morrison, K.M. (2013). The impact of obesity on quality of life. Best Pract. Res. Clin. Endocrinol. Metab., 27(2):139-146. https://doi.org/10.1016/ j.beem.2013.04.004.

Vasu, V.T.; Modi, H.; Thaikoottathil, J.V. and Gupta, S. (2005). Hypolipidaemic and antioxidant effect of Enicostemma littorale Blume aqueous extract in cholesterol fed rats. J. Ethnopharmacol., 101(1-3):277282. https://doi.org/10.1016/j.jep.2005.05.007

Vikram, N.K.; Pandey, R.M.; Misra, A.; Sharma, R.; Devi,J.R. and Khanna, N. (2003). Non-obese (body mass index $<25 \mathrm{~kg} / \mathrm{m} 2$ ) Asian Indians with normal waist circumference have high cardiovascular risk. Nutrition, 19(6), 503-509. https://doi.org/10.1016/S0899-9007(02)01083-3

Wierzbicki, A.S.; Mikhailidis, D.P.; Wray, R.; Schachter, M.; Cramb, R.; Simpson, W.G. and Byrne, C.B. (2003). Statin-fibrate combination therapy for hyperlipidaemia: A review. Curr. Med. Res. Opin., 19(3):155-168. https://doi.org/10.1185/030079903125001668

Wu, N.; Sarna, L.K.; Hwang, S.Y.; Zhu, Q.; Wang, P.; Siow, Y.L. and Karmin, O. (2013). Activation of 3-hydroxy-3-methylglutaryl coenzyme A (HMG-CoA) reductase during high fat diet feeding. Biochim. Biophys. Acta - Mol. Basis Dis., 1832(10), 1560-1568. https:// doi.org/10.1016/j.bbadis.2013.04.024

Laxmipriya Nampoothiri, Prashant Sudra, Arpi Dey, Shivani Dhadhal, Azazahemad A. Kureshi, Satyanshu Kumar, 